\title{
Integration of Mobile Ad Hoc Networks in 4G Networks
}

\author{
Nupur Soni \\ Assistant Professor, School of Computer Applications, Babu Banarsi Das University, Lucknow, India
}

\begin{abstract}
This Fourth-generation wireless networks may require an integration of mobile ad hoc networks (MANET) into external network to enhance the flexibility of the communication and roaming. This phenomenon is well-suited for commercial and military applications which yield additional benefit of roaming. However, integration of MANET with external network poses a serious security challenge for communication because of open and distributed nature of the ad hoc network. This paper discusses about the $4 \mathrm{G}$ network architecture, its challenges and finally the use of manet in $4 \mathrm{G}$ networks.
\end{abstract}

Keywords: internetworking, MANET, multimode end user terminal, QoS.

\section{INTRODUCTION}

This Before 3G was deployed all over the world, the idea of technology beyond 3G started evolving. This idea was beyond the imagination of ordinary mobile user promising "connect anytime, anyhow, anywhere" [1]. This ubiquitous network access will be achieved by seamlessly integrating the available and new networks using a core IP based network layer. This vision is called as the 4th generation of communication (4G).The existence of $4 \mathrm{G}$ Networks in today's technology-driven society is important indicators of advancement and change. 4G, or Fourth Generation networks, are designed to facilitate improved wireless capabilities, network speeds, and visual technologies. It is anticipated that as these networks continue to thrive, the demand for advanced related technologies will also grow, thereby creating new alternatives for savvy technology users to exceed their desired expectations. Like $4 \mathrm{G}$ project, MANET (Mobile Ad-hoc Network) is also in developing stage. Internet Engineering Task Force is working on routing techniques for MANET. Due to infrastructure less and self organizing nature of MANET, this network can be used in m- Governance. We introduce the term MANET@MGOV to describe the possible situations where MANET can be used in $\mathrm{m}$ - Governance. Emerging wireless technologies are expected to significantly influence design and implementation of MANET in $\mathrm{m}$ - Governance environment. As 4G provides the concept of convergence, the future wireless networks will take help of each other to provide anywhere, anytime, anyhow connectivity. Thus many networks can overcome their loopholes by converging and using the services of other networks to solve the constraints of MANET@MGOV.4G implemented with MANET which results 4GMANET@MGOV concept. (Kaur,B.Feb 2010).

\section{ARCHITECTURE OF 4G}

Figure 1 shows the proposed layered/level Architecture of 4G network elucidated in [2].

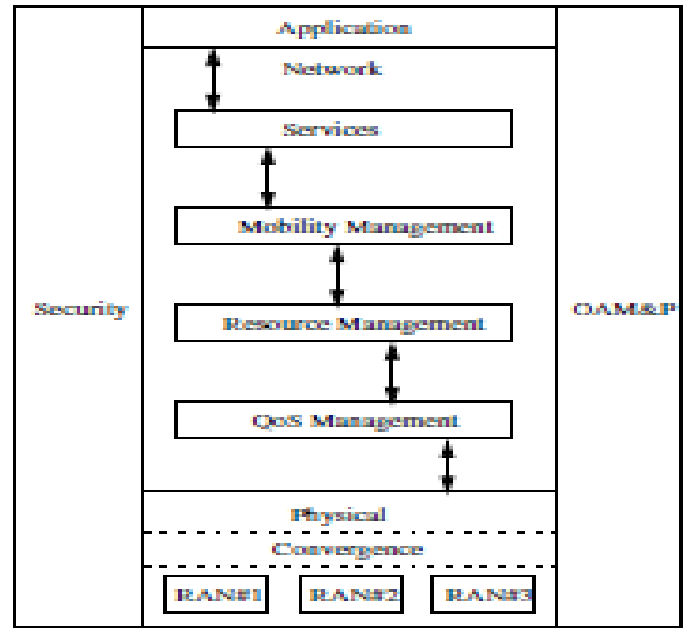

Fig I: Layered 4G architecture 
This architecture fulfills the basic requirement of servicing the standalone and mobile subscribers on an "anytime, anywhere, anyhow" basis in dynamic network conditions. The architecture is based on Internet Protocol version 6 (IPV6) which operates at the transport layer enabling seamless communication across various heterogeneous networks and based on the key factors such as mobility, Quality of Service (QoS) and efficient resource management schemes. The functionalities provided by each layer and module can be described as follows:

\section{II- A. Application}

This layer is composed of various third party applications which provide value added services to its subscribers.

Network

This layer consists of various sub layers described as follows:

$\square \square$ Services

This layer manages the interaction between various value-added services and networks.

$\square \square$ Mobility Management

This layer provides quality and uniform services to the mobile/stationary terminal across various heterogeneous networks. It provides features of low handover latency and packet loss during the provision of real-time and non-real time services to the end user moving across different networks. To achieve this, it performs tasks such as binding update (updating the care-off address of the mobile user), location management, common control signaling (signaling required to perform wireless network discovery), address assignment, handover control mechanism and so forth.

$\square \square$ Resource Management

This layer incorporates the functionalities of allocation, de-allocation and reallocation of the network resources which are acquired during the communication sessions within the same or different network domains. This activity is performed during or before the communication activity. This layer also performs the task of congestion control, packet scheduling and packet classification.

$\square \square$ Quality of service (QoS) management

This layer provides best optimal utilization of the available resources. In scenarios where the network resources are limited it provides an option to the applications to choose between high overall throughput and low end-to-end delay. It provides the best trade-off mechanisms depending on the application's preference. It encompasses several activities such as link utilization control, bandwidth control and so forth.

\section{II- B. Physical}

This layer consist of the core IPV6 network of 4G and other heterogeneous access networks such as GSM (Global System for Mobile communications), CDMA( Code Division Multiple Access) and WLAN in their physical view. This layer is composed of two sub-layers namely:

$\square$ Convergence layer

This layer provides common control signaling mechanism across the core and other heterogeneous networks at the physical level. It also allows different radio access networks to transparently use the independent network services such as mobility management, resource management and QoS management.

\section{Different RAN}

This layer consists of several radio access networks communicating with each other at the physical level. Operation, Administration, Maintenance and Provisioning

This layer spans across all the layers of the network architecture and provides the functionalities of network controlling, network monitoring and fault detection. It also maintains the repudiation between various services and resources of several heterogeneous and core networks.

\section{II-C Security}

This layer also branches across all the layers of the $4 \mathrm{G}$ network architecture which perform the function of authentication, authorization, encryption, establishment and implementation of service policy agreement between the various vendors.

\section{NEW CHALLENGES}

III- A. Security and Privacy

In the development of $4 \mathrm{G}$ Networks, security measures must be established that enable data transmission to be as safe as possible. Specifically, "The 4G core addresses mobility, security, and QoS through reuse of existing mechanisms while still trying to work on some mobility and handover issues" [3]. Therefore, it is necessary for the organization to develop an effective series of tools that support maximum $4 \mathrm{G}$ security measures as a means of protecting data that is transmitted across the network from hackers and other security violations. Because of the nature of the 4G network, there is an increased likelihood of security attacks, and therefore, multiple levels of security, including increased 
requirements for authentication, will be necessary to protect data and information that is transmitted across the network [3].

One of the main goals of G4 networks is to blanket very wide geographic area with seamless service. Obviously, smaller local area networks will run different operating systems. The heterogeneity of these wireless networks exchanging different types of data complicates the security and privacy issues. Furthermore, the encryption and decryption methods being used for $3 \mathrm{G}$ networks are not appropriate for $4 \mathrm{G}$ networks as new devices and services are introduced for the first time in $4 \mathrm{G}$ networks. To overcome these security and privacy issues, two approaches can be followed. The first is to modify the existing security and privacy methods so that they will be applicable to heterogeneous 4G networks. Another approach is to develop new dynamic reconfigurable, adaptive, and lightweight mechanisms whenever the currently utilized methods cannot be adapted to $4 \mathrm{G}$ networks [14].

\section{III- B. Quality of Service}

With respect to network quality, many telecommunications providers are promising that there will be enhanced connectivity, and the quality of data that is transmitted across the network will be of the highest possible quality, as in the case of Ericsson's 4G Network for TeliaSonera [7]. The company promises that "The new 4G network will do for broadband what mobile telephony did for voice. With real-time performance, and about 10 times higher data rates compared to today's mobile broadband networks, consumers can always be connected, even on the move" [7]. As a result, it is important for providers to develop an effective approach to the $4 \mathrm{G}$ Network that will enhance quality, provide effective security measures, and will ensure that all users are provided with extensive alternatives for downloading video, music, and picture files without delays.

The main challenge that $4 \mathrm{G}$ networks are facing is integrating non-IP-based and IP-based devices. It is known that devices that are not IP address based are generally used for services such as VoIP. On the other hand, devices that are IP address based are used for data delivery. 4G networks will serve both types of devices. Consequently, integrating the mechanisms of providing services to both non-IP-based as well as IP-based devices is one of key challenges $4 \mathrm{G}$ networks have to address $[17,19]$.

\section{III- C. Complex Architecture}

III (III-A)- Multimode End-User Terminals

To reduce operating costs, devices that operate on $4 \mathrm{G}$ networks should have the capability to operate in different networks. This will not only reduce the operating cost but will also simplify design problems and will reduce power consumption. However, accessing different mobile and wireless networks simultaneously is one of the major issues $4 \mathrm{G}$ networks have been addressing. One mechanism that has been proposed to handle this problem is termed "multi-mode devices". This mechanism can be achieved through a software radio that allows the end-user device to adapt itself to various wireless interfaces of the networks.

III (III-B) System Discovery and Selection

Due to the heterogeneity of $4 \mathrm{G}$ networks, wireless devices have to process signals sent from different systems, discover available services, and connect to appropriate service providers. Various service providers have their own protocols which can be incompatible with each other as well as with the user's device. This issue may complicate the process of selecting the most appropriate technology based on the time, place and service provided, and thus, may affect the Quality of service provided to the end user.

One solution to resolve this issue is called "System-initiated discoveries". This mechanism allows automatic download of software modules based on the wireless system the user is connected to [12]. Another approach to handle this problem is based overlay networks. In such case, the end-user device is connected to different networks through an overlay network.

\section{III (III-C) Service and Billing}

Managing user accounts and billing them has become much more complicated with $4 \mathrm{G}$ networks. This is mainly due to heterogeneity of $4 \mathrm{G}$ networks and the frequent interaction of service providers. The research community addressed this concern and proposed several frameworks to handle the customers' billing and user account information $[8,9]$.

\section{MANET EVOLUTION}

Historically, mobile ad hoc networks have primarily been used for tactical network related applications to improve battlefield communications/ survivability. The dynamic nature of military operations means that military cannot rely on access to a fixed pre-placed communication infrastructure in battlefield. Pure wireless communication also has limitation in that radio signals are subject to interference and radio frequency higher than $100 \mathrm{MHz}$ rarely propagate beyond line of sight (LOS) [10]. Mobile ad hoc network creates a suitable framework to address these issues by 


\section{International Journal of Advanced Research in Computer and Communication Engineering} ISO 3297:2007 Certified

Vol. 6, Issue 6, June 2017

providing a multi-hop wireless network without pre-placed infrastructure and connectivity beyond LOS. Early ad hoc networking applications can be traced back to the DARPA Packet Radio Network (PRNet) project in 1972 [10], which was primarily inspired by the efficiency of the packet switching technology, such as bandwidth sharing and storeandforward routing, and its possible application in mobile wireless environment. PRNet features a distributed architecture consisting of network of broadcast radios with minimal central control; a combination of Aloha and CSMA channel access protocols are used to support the dynamic sharing of the broadcast radio channel. In addition, by using multi-hop store-and-forward routing techniques, the radio coverage limitation is removed, which effectively enables multi-user communication within a very large geographic area. Survivable Radio Networks (SURAN) were developed by DARPA in 1983 to address main issues in PRNet, in the areas of network scalability, security, processing capability and energy management. The main objectives were to develop network algorithms to support a network that can scale to tens of thousands of nodes and withstand security attacks, as well as use small, low-cost, low-power radios that could support sophisticated packet radio protocols [10]. This effort results in the design of Low-cost Packet Radio (LPR) technology in 1987 [11], which features a digitally controlled DS spread-spectrum radio with an integrated Intel 8086 microprocessor-based packet switch. In addition, a family of advanced network management protocols was developed, and hierarchical network topology based on dynamic clustering is used to support network scalability. Other improvements in radio adaptability, security, and increased capacity are achieved through management of spreading keys [12]. Towards late 1980s and early 1990s, the growth of the Internet infrastructure and the microcomputer revolution made the initial packet radio network ideas more applicable and feasible [11]. To leverage the global information infrastructure into the mobile wireless environment, DoD initiated DARPA Global Mobile (GloMo) Information Systems program in 1994 [12], which aimed to support Ethernet-type multimedia connectivity any time, anywhere among wireless devices. Several networking designs were explored; for example Wireless Internet Gateways (WINGs) at UCSC deploys a flat peer-to-peer network architecture, while Multimedia Mobile Wireless Network (MMWN) project from GTE Internetworking uses a hierarchical network architecture that is based on clustering techniques. Tactical Internet (TI) implemented by US Army at 1997 is by far the largest-scale implementation of mobile wireless multi-hop packet radio network [11]. Direct-sequence spread-spectrum, time division multiple access radio is used with data rates in the tens of kilobits per second ranges, while modified commercial Internet protocols are used for networking among nodes. It reinforces the perception that commercial wireline protocols were not good at coping with topology changes, as well as low data rate, and high bit error rate wireless links [13].

In 1999, Extending the Littoral Battle-space Advanced Concept Technology Demonstration (ELB ACTD) was another MANET deployment exploration to demonstrate the feasibility of Marine Corps war fighting concepts that require overthe- horizon $(\mathrm{OTH})$ communications from ships at sea to Marines on land via an aerial relay. Approximately 20 nodes were configured for the network, Lucent_s WaveLAN and VRC-99A were used to build the access and backbone network connections. The ELB ACTD was successful in demonstrating the use of aerial relays for connecting users beyond LOS. In the middle of 1990, with the definition of standards (e.g., IEEE 802.11 [14]), commercial radio technologies have begun to appear on the market, and the wireless research community became aware of the great commercial potential and advantages of mobile ad hoc networking outside the military domain. Most of the existing ad hoc networks outside the military arena have been developed in the academic environment, but recently commercially oriented solutions started to appear (see, e.g., MeshNetworks 1 and SPANworks 2).

\section{AD HOC NETWORKING ISSUES}

In general, mobile ad hoc networks are formed dynamically by an autonomous system of mobile nodes that are connected via wireless links without using the existing network infrastructure or centralized administration. The nodes are free to move randomly and organize themselves arbitrarily; thus, the network_s wireless topology may change rapidly and unpredictably. Such a network may operate in a standalone fashion, or may be connected to the larger Internet. Mobile ad hoc networks are infrastructure-less networks since they do not require any fixed infrastructure, such as a base station, for their operation. In general, routes between nodes in an ad hoc network may include multiple hops, and hence it is appropriate to call such networks as "multi-hop wireless ad hoc networks". Each node will be able to communicate directly with any other node that resides within its transmission range. For communicating with nodes that reside beyond this range, the node needs to use intermediate nodes to relay the messages hop by hop. The ad hoc networks flexibility and convenience do come at a price. Ad hoc wireless networks inherit the traditional problems of wireless communications and wireless networking [14]: the wireless medium has neither absolute, nor readily observable boundaries outside of which stations are known to be unable to receive network frames;

- the channel is unprotected from outside signals;

- the wireless medium is significantly less reliable than wired media;

- the channel has time-varying and asymmetric propagation properties;

- hidden-terminal and exposed-terminal phenomena may occur. 
To these problems and complexities, the multihop nature, and the lack of fixed infrastructure add a number of characteristics, complexities, and design constraints that are specific to ad hoc networking [16, 17]: Autonomous and infrastructure-less. MANET does not depend on any established infrastructure or centralized administration. Each node operates in distributed peer-to-peer mode, acts as an independent router and generates independent data. Network management has to be distributed across different nodes, which brings added difficulty in fault detection and management.

Multi-hop routing. No default router available, every node acts as a router and forwards each others_packets to enable information sharing between mobile hosts. Dynamically changing network topologies. In mobile ad hoc networks, because nodes can move arbitrarily, the network topology, which is typically multi-hop, can change frequently and unpredictably, resulting in route changes, frequent network partitions, and possibly packet losses. Variation in link and node capabilities. Each node may be equipped with one or more radio interfaces that have varying transmission/receiving capabilities and operate across different frequency bands $[18,19]$. This heterogeneity in node radio capabilities can result in possibly asymmetric links. In addition, each mobile node might have a different software/hardware configuration, resulting in variability in processing capabilities. Designing network protocols and algorithms for this heterogeneous network can be complex, requiring dynamic adaptation to the changing conditions (power and channel conditions, traffic load/distribution variations, congestion, etc.). Energy constrained operation. Because batteries carried by each mobile node have limited power supply, processing power is limited, which in turn limits services and applications that can be supported by each node. This becomes a bigger issue in mobile ad hoc networks because, as each node is acting as both an end system and a router at the same time, additional energy is required to forward packets from other nodes. Network scalability. Currently, popular network management algorithms were mostly designed to work on fixed or relatively small wireless networks. Many mobile ad hoc network applications involve large networks with tens of thousands of nodes, as found for example, in sensor networks and tactical networks [11]. Scalability is critical to the successful deployment of these networks. The steps toward a large network consisting of nodes with limited resources are not straightforward, and present many challenges that are still to be solved in areas such as: addressing, routing, location management, configuration management, interoperability, security, high capacity wireless technologies, etc.

\section{4G AND AD HOC NETWORKING}

A major goal toward the $4 \mathrm{G}$ Wireless evolution is the providing of pervasive computing environments that can seamlessly and ubiquitously support users in accomplishing their tasks, in accessing information or communicating with other users at anytime, anywhere, and from any device [20]. In this environment, computers get pushed further into background; computing power and network connectivity are embedded in virtually every device to bring computation to users, no matter where they are, or under what circumstances they work. These devices personalize themselves in our presence to find the information or software we need.The new trend is to help users in the tasks of everyday life by exploiting technologies and infrastructures hidden in the environment, without requiring any major change in the users_behavior.

This new philosophy is the basis of the Ambient Intelligence concept [21]. The objective of ambient intelligence is the integration of digital devices and networks into the everyday environment, rendering accessible, through easy and "natural" interactions, a multitude of services and applications. Ambient intelligence places the user at the center of the information society. This view heavily relies on $4 \mathrm{G}$ wireless and mobile communications. $4 \mathrm{G}$ is all about an integrated, global network, based on an open systems approach. Integrating different types of wireless networks with wire-line backbone network seamlessly, and convergence of voice, multimedia and data traffic over a single IP-based core network are the main foci of 4G. With the availability of ultra-high bandwidth of up to $100 \mathrm{Mbps}$, multimedia services can be supported efficiently; ubiquitous computing is enabled with enhanced system mobility and portability support, and location- based services are all expected. Fig. 2 illustrates the networks and components within $4 \mathrm{G}$ network architecture. Network Integration. 4G networks are touted as hybrid broadband networks that integrate different network topologies and platforms. In Fig.2 the overlapping of different network boundaries represents the integration of different types of networks in 4G. There are two levels of integration. First is the integration of heterogeneous wireless networks with varying transmission characteristics such as Wireless LAN, WAN, PAN, as well as mobile ad hoc networks. At the second level we find the integration of wireless networks with the fixed network backbone infrastructure, the Internet, and PSTN. Much work remains to enable a seamless integration, for example that can extend IP to support mobile network devices. All IP Networks. 4G starts with the assumption that future networks will be entirely packet-switched, using protocols evolved from those in use in today_s Internet [22]. An all IP-based 4G wireless network has intrinsic advantages over its predecessors. IP is compatible with, and independent of, the actual radio access technology, this means that the core $4 \mathrm{G}$ network can be designed and evolves independently from access networks. Using IPbased core network also means the immediate tapping of the rich protocol suites and services 
already available, for example, voice and data convergence, can be supported by using readily available VoIP set of protocols such as MEGACOP, MGCP, SIP, H.323, SCTP, etc. Finally the converged all-IP wireless core networks will be packet based and support packetized voice and multimedia on top of data. This evolution is expected to greatly simplify the network and to reduce costs for maintaining separate networks, for different traffic types.

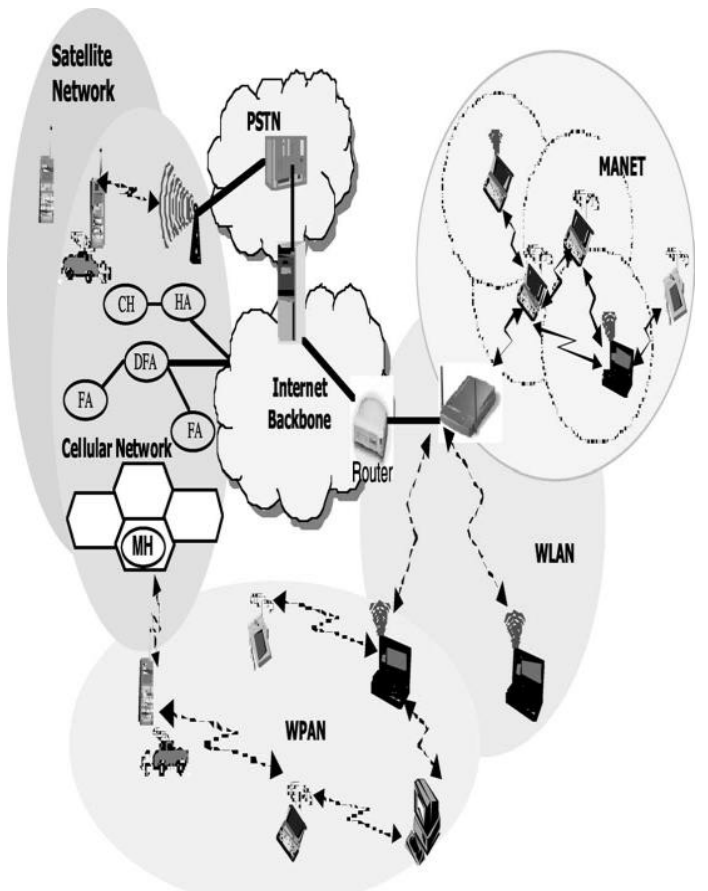

Fig.-II. 4G networks with MANET.

Lower Cost and Higher Efficiency. 4G IP-based systems will be cheaper and more efficient than 3G. Firstly, equipment costs are expected to be four to ten times lower than equivalent circuitswitched equipment for $2 \mathrm{G}$ and $3 \mathrm{G}$ wireless infrastructures. An open converged IP wireless environment further reduces costs for network build-out and maintenance. There will be no need to purchase extra spectrum as $2 \mathrm{G} / 3 \mathrm{G}$ spectrum can be reused in $4 \mathrm{G}$, and much of spectrum needed by WLAN and WPAN is public and does not require a license. Ultra-High Speed and Multimedia Applications. 4G systems aim to provide ultra-high transmission speed of up to $100 \mathrm{Mbps}$, 50 times faster than those in 3G networks. This leap in provided bandwidth will enable high-bandwidth wireless services, allowing users to watch $\mathrm{TV}$, listen to the music, browse Internet, access business programs,perform real-time video streaming and other multimedia-oriented applications, like E-Commerce, as if sitting in home or office. Location Intelligence. To support ubiquitous computing requirements, 4G terminals need to be more intelligent in terms of user_s locations and service needs, including recognizing and being adaptive to user_s changing geographical positions, as well as offering locationbased services [23].

Anytime anywhere requires intelligent use of location information, and the embedding of the information into various applications. Possible Location Based Services include finding nearest service providers, such as restaurant or cinema; searching for special offers within an areas; warning of traffic or weather situations; sending an advertisement to a specific area; searching for other users; active badge systems, etc. Outdoor, wireless applications can use GPS to obtain location information. GPS is a satellite-based system that can provide easy, accurate positioning information almost anywhere on earth. Many GPS implementations are available, including integrating a GPS receiver into a mobile phone (GPS/ DGPS); or add fixed GPS receivers at regular intervals to obtain data to complement readings on phone (AGPS); or by using help from fixed base stations (E-OTD). These implementations provide different fix time and accuracy ranging from 50 to $125 \mathrm{~m}$. For indoor applications, since GPS signal cannot be received well inside the buildings, alternative technologies like Infrared, Ultrasound or Radio are being considered. Non-infrastructure-based MANET are expected to become an important part of the $4 \mathrm{G}$ architecture. An ad hoc mobile network is a transient network formed dynamically by a collection of (arbitrarily located) wireless mobile nodes without the use of existing network infrastructure, or centralized administration. Ad hoc networks are created, for example, when a group of people come together, and use wireless communications for some computer-based collaborative activities; this is also referred to as spontaneous networking [24]. In a MANET, the users_ mobile devices are the network, and they must cooperatively provide the functionality usually provided by the network infrastructure (e.g., routers, switches, servers). 
In a MANET, no infrastructure is required to enable information exchange among users_ mobile devices. We can envisage these devices as an evolution of current mobile phones, and emerging PDA_s equipped with wireless interfaces. The only external resource needed for their successful operation is the bandwidth, often the (unlicensed) ISM band. Nearby terminals can communicate directly by exploiting, for example, wireless LAN technologies. Devices that are not directly connected, communicate by forwarding their traffic via a sequence of intermediate devices. MANETs are gaining momentum because they help realizing network services for mobile users in areas with no preexisting communications infrastructure, or when the use of such infrastructure requires wireless extension $[25,26]$. Ad hoc nodes can also be connected to a fixed backbone network through a dedicated gateway device enabling IP networking services in the areas where Internet services are not available due to a lack of preinstalled infrastructure. All these advantages make ad hoc networking an attractive option in future wireless networks.

\section{CONCLUSION}

Ad hoc networking is at the center of the evolution towards the 4th generation wireless technology. Its intrinsic flexibility, ease of maintenance, lack of required infrastructure, auto-configuration, self administration capabilities, and significant costs advantages make it a prime candidate for becoming the stalwart technology for personal pervasive communication. The opportunity and importance of ad hoc networks is being increasingly recognized by both the research and industry community, as evidenced by the flood of research activities, as well as the almost exponential growth in the Wireless LANs and Bluetooth sectors.

MANET WG proposes a view of mobile ad hoc networks as an evolution of the Internet. This mainly implies an IPcentric view of the network, and the use of a layered architecture. Current research points out though that this choice may limit developing efficient solutions for MANET. Other promising directions have been identified [27]. The use of the IP protocol has two main advantages: it simplifies MANET interconnection to the Internet, and guarantees the independence from wireless technologies On the other hand, more efficient and lightweight solutions can be obtained, for example, by implementing routing solutions at lower layers [28,29]. Furthermore, masking lower layers_ characteristics may not to be useful in MANET. The layered paradigm has highly simplified Internet design, however when applied to ad hoc networks, it may result in poor performance as it prevents exploiting important interlayer dependencies in designing efficient ad hoc network functions. For example, from the energy management standpoint, power control and multiple antennas at the link layer are coupled with power control and scheduling at MAC layer, and with energy-constrained and delay-constrained routing at network layer

\section{REFERENCES}

[1] A. H. Khan, M. A. Qadeer, J. A. Ansari and S. Waheed. 4G as a Next Generation Wireless Network. Future Computer and Communication, 2009. ICFCC 2009. International Conference, April 2009, Page(s):334 - 338 [2] Kibria, M.R.; Mirchandani, V.; Jamalipour, A. A Consolidated Architecture for 4G/B3G Networks. Wireless Communications and Networking Conference, 2005, IEEE Volume 4, March 2005, Page(s):2406 $-2411$.

[3] Berardinelli, G.; Ruiz de Temino, L.A.; Frattasi, S.; Rahman, M.; Mogensen, P. OFDMA vs. SC-FDMA: performance comparison in local area imt-a scenarios. IEEE Wireless Communications, Volume 15, Issue 5, October 2008, Page(s):64-72.

[4] Goldsmith, A.; Jafar, S.A.; Jindal, N.; Vishwanath, S. Capacity Limits of MIMO Channels. Selected Areas in Communications, IEEE Journal Volume 21, Issue 5, June 2003, Page (s): $684-702$.

[5] Adachi, K.; Adachi, F.; Nakagawa, M. A Study on Channel Capacities of MC-CDMA MIMO and OFDM MIMO. Communication Systems, 2008. ICCS 2008. $11^{\text {th }}$ IEEE Singapore International Conference, Nov. 2008, Page(s):1384 - 1388.

[6] Xiaodong Wang. OFDM and Its Application to 4G. Wireless and Optical Communications, 2005. 14th Annual WOCC 2005. International Conference, April 2005 Page(s):69.

[7] Mitola, J.; III E-Systems, Fairfax, VA. Software Radios Survey, Critical Evaluation and Future Directions. Aerospace and Electronic Systems Magazine, IEEE, Volume 8, Issue 4, April 1993, Page(s):25 - 36 [8] Sharony, J. Introduction of Wireless Theory and Application. IEEE LI, November 2006.

[9] Suk Yu Hui, Kai Hau Yeung. Challenges in the Migration to 4G Mobile Systems. Communications Magazine, IEEE Volume 41, Issue 12, Dec. 2003, Page(s):54 - 59

[10] James A. Freebersyser, Barry Leiner, A DoD perspective on mobile ad hoc networks, in: Charles E. Perkins (Ed.), Ad Hoc Networking, Addison Wesley, Reading, MA, 2001, pp. 29-51.

[11] W. Fifer, F. Bruno, The low-cost packet radio, Proceedings of the IEEE 75 (1) (1987) 33-42.

[12] N. Shacham, J. Westcott, Future directions in packet radio architectures and protocols, Proceedings of the IEEE 75 (1) (1987) 83-99.

[13] J. Strater, B. Wollman, OSPF Modeling and Test Results and Recommendations, Mitre Technical Report 96W0000017, Xerox Office Products Division, March 1996.

[14] IEEE standard for Wireless LAN- Medium Access Control and Physical Layer Specification, P802.11, November 1997. See also.

[15] IEEE P802.11/D10, January 14, 1999

[16] M.S. Corson, J.P. Maker, J.H. Cernicione, Internet-based mobile ad hoc networking, IEEE Internet Computing 3 (4) (1999) 63-70.

[17] M.S. Corson, J.P. Maker, J.H. Cernicione, Internet-based mobile ad hoc networking, IEEE Internet Computing 3

(4) (1999) 63-70.

[18] I. Chlamtac, A. Lerner, Link allocation in mobile radio networks with noisy channel, in: IEEE INFOCOM, Bar

Harbour, FL, April 1986.

[19] I. Chlamtac, A. Lerner, Fair algorithms for maximal link activiation in multi-hop radio networks, IEEE Transactions on Communications COM35 (7) (1987). 
[20] M. Weiser, The Computer for the Twenty-First Century, Scientific American, 1991

[21] J. Ahola, Ambient Intelligence, ERCIM (European Research Consortium for Information and Mathematics) NEWS, N. 47, October 2001.

[22] Y. Bing Lin, Y.R. Huang, A. Pang, I. Chlamtac, All-IP approach for third generation mobile networks, IEEE Network Magazine, in press.

[23] S. Basagni, I. Chlamtac, A.V.R. Syrotiuk, Location aware one-to-many communication in mobile multi-hop wireless networks, in: Proceedings of the IEEE Vehicular Technology (VTC), Tokyo, Japan, May 2000.

[24] L. Feeney, B. Ahlgren, A. Westerlund, Spontaneous networking: an application-oriented approach to ad hoc networking, IEEE Communications Magazine (2001)

[25] M.S. Corson, J.P. Maker, J.H. Cernicione, Internet-based mobile ad hoc networking, IEEE Internet Computing 3

(4) (1999) 63-70.

[26] S. Giordano, Mobile ad-hoc networks, in: I. Stojmenovic (Ed.), Handbook of Wireless Networks and Mobile Computing, Wiley, New York, 2002

[27] A.J. Goldsmith, S.B. Wicker, Design challenges for energy-constrained ad hoc wireless networks, IEEE Wireless Communications 9 (4) (2002) $8-27$.

[28] A. Acharya, A. Misra, S. Bensal, A label-switching packet forwarding architecture for multi-hop wireless LANs, in: M. Conti, D. Raychaudhuri (Eds.), Proceedings of the ACM Workshop on Mobile Multimedia (WoWMoM 2002), Atlanta, GA, September 28, 2002.

[29] C. Tschudin, R. Gold, LUNAR: Lightway Underlay Network Ad Hoc Routing. Available from <http://www.docs.uu.se/docs/research/projects/selnet/lunar/lunar.pdf>.

[30] Ya Xu, John Heidemann, Deborah Estrin, Adaptive energy conserving routing for multihop ad hoc networks, Technical Report 527, USC/Information Sciences Institute, October 2000.

[31] Ya Xu, John Heidemann, Deborah Estrin, Geographyinformed energy conservation for ad hoc routing, in: Proceedings of 7th Annual International Conference on Mobile Computing and Networking, July 2001, pp. 70-84.

[32] S. Xu, T. Saadawi, Does the IEEE 802.11 MAC protocol work well in multihop wireless ad hoc networks? IEEE Communication Magazine 39 (6) (2001) 130-137.

[33] S. Xu, T. Saadawi, Revealing the problems with 802.11 MAC protocol in multi-hop wireless networks, Computer Networks 38 (4) (2002).

[34] H. Xiao, W.K.G. Seah, A. Lo, K.C. Chua, A flexible quality of service model for mobile ad-hoc networks, IEEE VTC2000-spring, Tokyo, Japan, May 2000.

[35] Jungkeun Yoon, Mingyan Liu, Brian Noble, Random waypoint considered harmful, in: Proceedings of INFOCOM

2003, San Francisco, April 2003.

[36] Xin Yuan, Arif Saifee, Path selection methods for localized quality of service routing, in: Proceedings of IEEE ICCCN2001, Scottsdale, AZ, October, 2001. 